\title{
Experimental Study of Flow Division in a Smooth Circular Culvert
} Yu Han ${ }^{1}$,Liu-chao Qiu ${ }^{2, *}$, Xiao-dong Liu ${ }^{3}$ and Shu-qingYang ${ }^{4}$

\author{
${ }^{1}$ Collage of Water Resources \& Civil Engineering, China Agricultural University, Beijing 100083,
} China. ${ }^{2}$ Collage of Water Resources \& Civil Engineering, China Agricultural University, Beijing 100083,
China.Email:

${ }^{3}$ Collage of Water Resources \& Civil Engineering, China Agricultural University, Beijing 100083, China.Email:

${ }^{4}$ School of Civil, Mining \& Environmental Engineering, University of Wollongong, NSW 2522, Australia.

yh916@uowmail.edu.au ${ }^{1}, 2$ qiuliuchao@cau.edu.cn, ${ }^{3} 2427817067 @ q q . c o m,$, shuqing@uow.edu.au.

${ }^{*}$ Corresponding author

Keywords: Flow Division, Turbulence, Velocity Distribution, LDA,Reynolds Shear Stress.

\begin{abstract}
The idea of partitioning the flow has been widely used by hydraulic researchers and engineers. Currently, researchers claim that their models for the division lines are valid and yield good agreement with the experimental data from a channel flow that has a flat bed. It is not clear whether their models are still effective to model the division lines when the channel bed is curved. The aim of this paper is to examine the existence of division lines by analysing the distribution of mean velocity in a smooth culvert. The experiments reported here document a sufficient fact that it possible to observe the division lines from the velocity distribution. The method used to test the existence of the division line is to determine the normal distance from a boundary $L_{n}$, based on the local boundary shear stress. The model from Yang and Lim (YLM) is selected for considerations in this comparison because other models were unable to produce the theoretical division lines in these cases. The division lines obtained from YLM are also plotted for various experimental flows to demonstrate the relationship between the experimental and theoretical division lines. This experimental analytical method developed for division lines may be extended to any arbitrary channels in other channel flows based on the results of the distribution of velocity.
\end{abstract}

\section{Introduction}

Flow partitioning theory has been widely used in open channel flows by researchers such as Keulegan (1938), Einstein (1942), Yang and Lim (1997), Chien and Wan (1999), Guo and Julien (2005). Several mathematical treatments have been proposed to divide the cross section of a rectangular or trapezoidal channel into subsections (!!! INVALID CITATION !!!), but only a handful of experimental research has been conducted where these mathematical arguments were compiled, so the physical meaning of division lines is not understood very well (Yang, 2005). As for the division lines, it is easy to infer that the flow region is dividable if the shear stress measured along these lines is zero, but achieving accurate Reynolds shear stress measurements is very difficult relative to the mean velocity measurements. Currently, there are no specially designed experiments to verify them or the models, not to mention whether the existence of division lines can be inferred from the mean velocity profiles.

Although the concept of flow partition is widely accepted, an extensive literature review reveals that there is no research work on flow partitioning in a culvert. The primary motivation for partitioning the flow in the cross section of a smooth open channel stemmed from the flow of wastewater in sewers and drainage system. In these cases, the local shear stress needs to be determined in order to prevent sewer erosion (!!! INVALID CITATION !!!). The study of flow partitioning in a culvert is also significant in various engineering applications such as estimating the 
sidewall affection and stability in culverts and storm water pipes. It is widely believed that the theory of flow partitioning is an effective mathematical tool to simplify the hydraulic calculation without any physical meaning, and the practice shows that such treatment can significantly enhance the accuracy for estimation of bed load transport, the bed form resistance, and the pollutant transport. Therefore, it is necessary to experimentally ascertain the existence of the division lines in a channel flow. Thus, it is natural to ask whether a 3-D flow in culverts can be divided into separate divisions as trapezoidal or triangles and how the division lines within a concave cross-section be determined. The purpose of this paper is to develop a feasible method for detecting the existence of division lines in a channel flow by analysing the distribution of mean velocity in a channel, and if they exist, should they be represented in culverts.

\section{Determining the Division Line from Experiments}

Firstly, Yang (2006) proposed that the zero shear stress in a flow is a direct and straight forward method of identifying the existence of division lines, i.e., the shear stress on the division lines should be equal to zero if the flow region can be divided, which means that no turbulent energy is transferred across them, i.e.,

$$
\tau=\rho\left(v+v_{T}\right) \frac{\partial u}{\partial t}=0
$$

Where $\rho^{\rho}$ is fluid density, and $\mathrm{u}$ is the velocity component in a stream wise direction. $\mathrm{t}$ is the tangential direction of the division line, $v$ is kinematic viscosity, and $v_{T}$ is eddy viscosity, often called the 'apparent' or 'virtual' viscosity. If a flow region is dividable, then the shear stress along the division line as defined in Eq. (1) must be zero. Furthermore, inferring that dividing lines from the distribution of velocity exist could also be a useful and interesting topic because it could apply to any shape of cross sectional flow.

The present investigations are directed toward the structure of turbulent flow in a uniform open channel in which the channel bedforms have been fabricated as concave bed. Throughout the experimental process, the water velocities were measured using a Dantec two component LDA system. No calibration is necessary because the relevant relationship between the velocity and the Doppler frequency can be determined quite accurately from theory. This paper therefore investigates whether a 3-D flow region can be divided by analysing the measured mean velocity data. This method may help practicing engineers discern the existence of a division line from a measured velocity distribution. Furthermore, inferring the existence of division lines from the normal distance Ln from a boundary based on the local boundary shear stress was also considered in this study. Based on this assumption, a new method to determine the location of the division lines has been established.

Thus, it would be better to go back to the general form of the Boussineq eddy viscosity model:

$$
-\overline{u^{\prime} v^{\prime}}=v_{T} \frac{d u}{d y}
$$

Where, $-\overline{u^{\prime} v^{\prime}}$ denotes the Reynolds stress, $d u / d y$ stands for the streamwise velocity (u) gradient with respect to the wall normal direction (y). For wall-bounded turbulent flows, the eddy viscosity $v_{T}$ must vary with the distance from the wall. Prandtl proposed a 'mixing length' theory to express the eddy viscosity:

$$
v_{T}=l_{m}^{2}\left|\frac{d u}{d y}\right| .
$$


In the region near a wall, the shear stress is assumed to be constant and

$$
-\overline{\rho u^{\prime} v^{\prime}}=\rho l_{m}^{2}\left|\frac{d u}{d y}\right| \frac{d u}{d y}=\rho u_{*}^{2} .
$$

By assuming that the mixing length $1 \mathrm{~m}$ is proportional to the distance to the boundary, $1 \mathrm{~m}=k \mathrm{y}$, herein $k$ is the von Karman constant. This value may be slightly different from the value of 0.4 obtained by Nikuradse (Cardoso et al., 1989, Smits et al., 2011). The integration of Eq. (3) with the boundary condition $\mathrm{y}=\mathrm{y} 0, \mathrm{u}=0$ (Yang et al., 2012), yields the logarithmic velocity profile from Eq. (4).

$$
\frac{u}{u_{* 1}}=\frac{1}{\kappa} \ln \frac{y}{y_{0}} .
$$

Where $U_{*_{1}}$ is a friction velocity. In Prandtl's 2-D flows theorem, the parameter y0 is the constant of integration that must be determined using the boundary condition, where it is expected that the parameter y0 in Eq. (5) should be a variable as well, using the velocity continuity condition y0 could be related to local velocity at the viscous sublayer, i.e.,

$$
y_{0}=\frac{v}{c u_{*_{\mathrm{s}}}} .
$$

Where $\mathrm{c}$ is the coefficient to be determined experimentally and $u_{*_{\mathrm{s}}}$ is denoted as the local friction velocity, which may be different from ${ }^{u_{* 1}}$.Then, by substituting the Eq. (6) into Eq. (5), the velocity profile normal to the boundary can be expressed as the classic log-law:

$$
\frac{u}{u_{* 1}}=\frac{1}{\kappa} \ln \frac{u_{*} y}{v}+\frac{1}{\kappa} \ln c .
$$

Eq. (7) is the most acceptable form as classic log law. In a pipe flow, Nikuradse's data (1932) confirms that $u_{*_{1}}=u_{*_{\mathrm{s}}}$. To examine whether $u_{*_{1}}=u_{*_{\mathrm{s}}}$ is universal, it is worth investigating how to determine the local shear velocity experimentally. Generally, it is clear that in a plot of velocity measured against log yn (normal direction) for every profile along the dashed lines in Fig. 1, the unknown ${ }^{u_{* 1}}$ can be evaluated from its slope (i.e., ${ }^{u_{*_{1}} / k}$ and the other unknown ${ }^{u_{*_{s}}}$ can be evaluated from the interception. In open channel flow, once the local shear velocities $u^{*} s$ are obtained from the velocity profiles then the characteristic distance of $\mathrm{Ln}$ from the division line to the boundary can be determined, i.e. $L_{n}=\mathrm{u}_{*_{s}}^{2} /(g S)$. Therefore, the upper boundary of the sub-region or division line can be inferred from the location of Ln.

\section{Experimental Setup}

Yang (1997) proposed that a log law gives a good description of the distribution of velocity in the centre region after introducing $\mathrm{u}^{*} 1=\sqrt{g \mathbf{R S}}$ and $\mathrm{u}^{*} \mathrm{~s}=\sqrt{g \mathbf{h S}}$, then the other question is whether this principle can be used for all the velocity profiles along the normal direction of the wetted perimeter in a circular channel? To test whether we can use two different shear velocities in the log-law in a circular channel, and how to divide the flow in terms of the distribution of velocity, 
data from research conduction by Knight and Sterling (2000) has been analysed in this study. Relevant experimental information is shown in Table 1.

The other experiments $(\mathrm{C} 1, \mathrm{C} 2$ and $\mathrm{C} 3)$ were undertaken in a large-scale flow loop at the University of Wollongong (UOW), Australia. The sidewalls and bed of the flume were constructed from $10 \mathrm{~mm}$ thick smooth glass that was $11 \mathrm{~m}$ long by $0.3 \mathrm{~m}$ wide by $0.45 \mathrm{~m}$ high, with a slope set to a value of about $0.1 \%$. A semi-pipe (radius $\mathrm{r}=150 \mathrm{~mm}$ ) was incorporated into the flume to manufacture a concave bed form, as shown in Fig.1. The main hydraulic parameters used in the experiments $\mathrm{C} 1, \mathrm{C} 2$ and $\mathrm{C} 3$ are listed in Table 1. The aim of the experimental program was to undertake a series of velocity distribution measurements with different $\mathrm{h} / \mathrm{r}$ values, and then use these measured velocities to test whether the circular flow region is valid by log-law and how to divide the cross section by analysing the distribution of velocity. In order to visualise the mean flow pattern across the section, the sampling mesh (Fig. 1) were extended to a complete rectangular size where unknown values at the extended points were extrapolated from the data collected using interpolation. The test section was located $6 \mathrm{~m}$ downstream from the entrance. Uniform flow was established within the flume prior to the collection of any experimental data. Three sampling locations at distances $1.0 \mathrm{~m}, 2.6 \mathrm{~m}$ and $4.0 \mathrm{~m}$ from the channel entrance respectively were chosen. From the data recorded at these locations by the Nixon Streamflo velocity meter, it was possible to visually analyse the development of uniform flow in the channel.

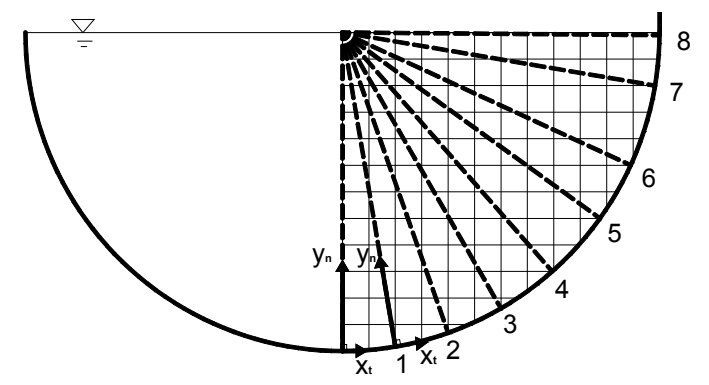

Fig 1 .Channel cross section and notation

Table 1. Summary of Main Hydraulic Parameters

\begin{tabular}{|c|c|c|c|c|c|c|c|}
\hline & $\mathrm{h} / \mathrm{r}$ & $\begin{array}{l}\mathrm{r} \\
\text { radius: [m] }\end{array}$ & $\begin{array}{l}\mathrm{h} \\
\text { water depth:[m] }\end{array}$ & $\mathrm{S}$ & $\begin{array}{l}\mathrm{Q} \\
{\left[\mathrm{m}^{3} / \mathrm{s}\right]}\end{array}$ & $\begin{array}{l}\mathrm{U} \\
{[\mathrm{m} / \mathrm{s}]}\end{array}$ & $\sqrt{g R S}$ \\
\hline \multirow{2}{*}{$\begin{array}{l}\text { Knight and } \\
\text { Sterling } \\
(2000)\end{array}$} & 0.67 & 0.122 & 0.081 & 0.001 & $5.36 \times 10^{-3}$ & 0.394 & 0.021 \\
\hline & 1.01 & 0.122 & 0.124 & 0.001 & $11.7 \times 10^{-3}$ & 0.493 & 0.024 \\
\hline $\mathrm{C} 1$ & 0.5 & 0.15 & 0.075 & 0.001 & 0.009 & 0.461 & 0.0241 \\
\hline $\mathrm{C} 2$ & 0.83 & 0.15 & 0.125 & 0.001 & 0.016 & 0.560 & 0.0263 \\
\hline $\mathrm{C} 3$ & 0.93 & 0.15 & 0.14 & 0.001 & 0.018 & 0.569 & 0.0268 \\
\hline
\end{tabular}

\section{Results and Analysis}

\section{Division Lines Inferred from the Velocity Profiles}

For the purpose of this current study, the log-law distribution of mean velocities by Knight and Sterling (2000) were plotted in Figs. 2 and 3. First, the velocity along each normal profile yn (Fig.1) as measured by Knight and Sterling (2000) was plotted in the form $u / \sqrt{g R S}$ versus log yn in Figs. 
2 and 3 to demonstrate that the velocity profile at different locations of $\operatorname{Pr} / \mathrm{P}$ cannot be represented very well. Here, Pr is the distance along the wetted perimeter which started at the right bank at the free surface. $\mathrm{P}$ denotes the half length of the wetted perimeter. Figs. 2 and 3 also suggest that to a certain extent, the velocity measured in the near wall region follows a straight line (solid and dashed lines in Figs. 2 and 3). It is interesting to note that all the straight lines shared the same slope but with different intercepts. Having an identical slope implies that $\mathrm{u}^{*} 1$ in Eq. (7) is constant as the mean shear velocity, but the different intercepts implies that the shear velocity $\mathrm{u}^{*} \mathrm{~s}$ in Eq. (7) is a variable. Thus, when the $u^{*}$ s from each straight line can be obtained the calculated results show that local shear velocity $\mathrm{u}^{*} \mathrm{~s}$ increases when $\mathrm{Pr} / \mathrm{P}$ increases.

Likewise, the distributions of mean velocity detected from the UOW circular channel $(\mathrm{C} 1, \mathrm{C} 2$ and C3) are also shown in dimensionless form in Figs. 4, 5 and 6 respectively, in terms of $u / u_{* 1}$ and $\log$ yn. The similar deviation is evidence that the velocity profiles for different vertical lines do not have a common intercept. It can also be clearly seen that the slope of each profile is quite similar, which means that the mean shear velocity ${ }^{u_{* 1}}$ used into the LHS of Eq. (7) is constant for different vertical locations. These findings arrive at the same results as Knight and Sterling (2000) data in that the variation of intercepts corresponding to each velocity profile that represent the shear velocity in RHS of Eq. (7) must be different. Likewise, once the local shear velocities $u_{*_{S}}$ are obtained from the velocity distributed for each velocity profile, then the distance ${ }^{L_{n}}$ can be determined from local shear, i.e., $L_{n}=u_{*_{s}}^{2} /(g S)$.Yang and Lim (1997) hypothesised that turbulent energy must be transported towards the boundary for dissipation through the minimum relative distance so the distance of Ln can be denoted as the energy transfer path. Hence, we can conclude that the distance Ln depicts the upper boundary of the sub-region or the division line presented as solid points in Figs. 4, 5 and 6. From this particular data set of Ln, it clearly illustrated the physical existence of division lines in experimental flow.

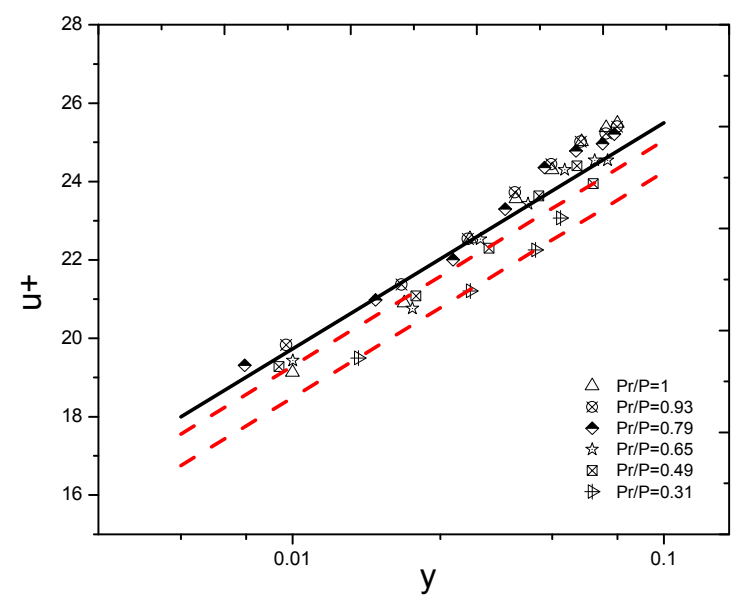

Fig 2. Measured velocity distribution for a semi-circular channel running partially full $\mathrm{h} / \mathrm{r}=0.67$ (after Knight and Sterling (2000) data) 


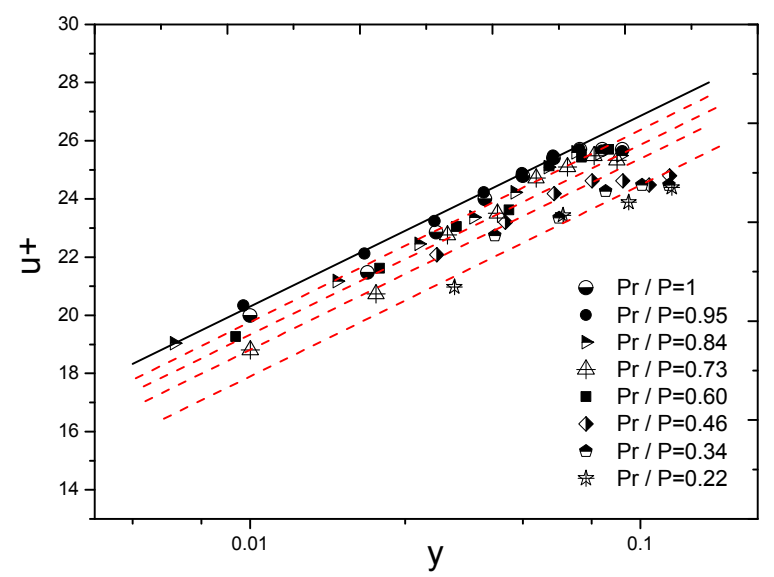

Fig 3. Measured velocity distribution for a semi-circular channel running partially full h/r=1 (after Knight and Sterling (2000) data)

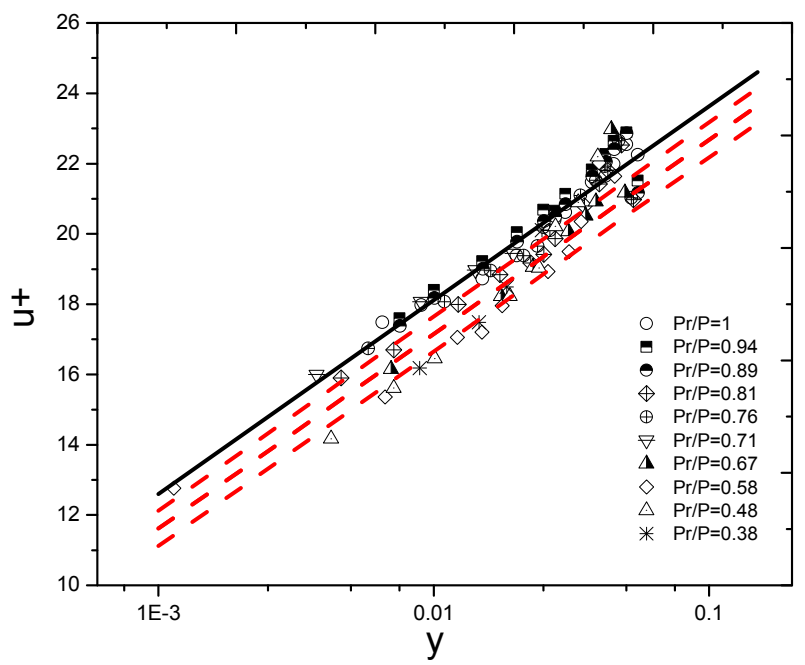

Fig 4. Measured velocity distribution for a semi-circular channel running partially full $\mathrm{h} / \mathrm{r}=0.5$

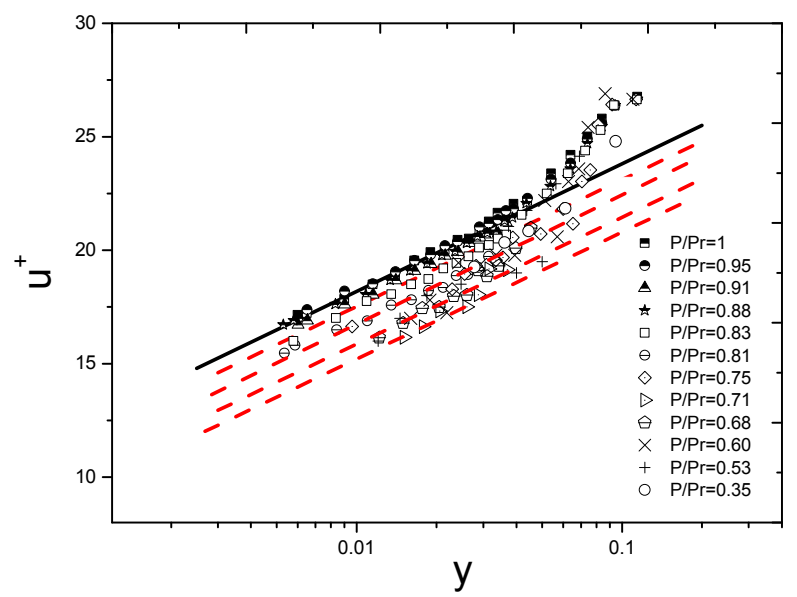

Fig 5. Measured velocity distribution for a semi-circular channel running partially full

$$
\mathrm{h} / \mathrm{r}=0.83
$$

The theoretical division lines obtained from YLM were also plotted (Fig. 7) to demonstrate the relationship between the experimental and theoretical division lines. Other models like DM, KM and GJM are unable to produce the division lines in these cases because they are not able to distinguish sidewall and bed in culverts. For any point in culvert flow field, there is only one normal line from that point to the curved boundary. Thus, according to the concept proposed in YLM, the 
surplus energy within the flow area will be transferred towards, and dissipated on the wetted perimeter along normal lines. Thus, the Reynolds shear stress increases linearly from zero at the division lines in the main flow region to the boundary shear stress, and the velocity increases logarithmically from zero at the boundary to its maximum value on the division lines. Hence, using the YLM model, the division line or zero shear stress line should be presented as the free surface line in channel flows. Therefore, the experimental results can be used to check whether YLM is accurate enough to express the measured division lines. A comparison of the experimental and the theoretical division lines are presented in Fig. 7.

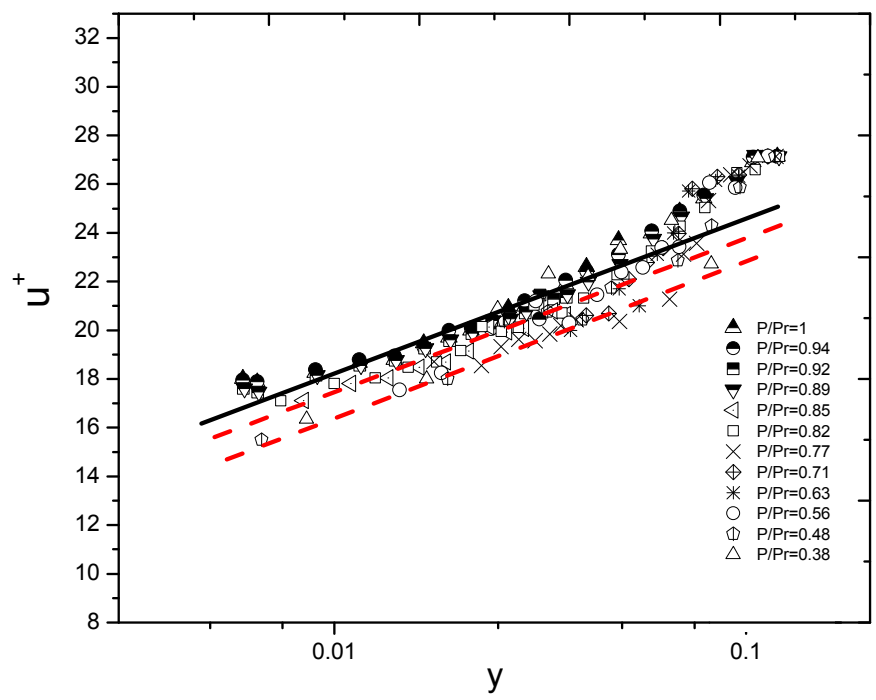

Fig 6. Measured velocity distribution for a semi-circular channel running partially full $\mathrm{h} / \mathrm{r}=0.93$

Fig. 7 shows the comparison between the division lines obtained by YLM and the measured result by $\mathrm{Ln}$. It is seen that the relative distance $(\mathrm{yn} / \mathrm{h})$ calculated from YLM agrees reasonably well the measured data $\mathrm{Ln} / \mathrm{h}$, even though noticeable small discrepancies exist in the region $(\mathrm{Pr} / \mathrm{P})$ from 0.5 to 0.8 . By using the YLM model, the division line should stand for the free surface line in channel flows. Therefore, it seems the division line determined from Ln in culvert experiments is quite close to the water surface. Thus, we can conclude that the real division line used in culvert study can approximately be considered as water surface line. To validate the accuracy of YLM, the relative error between YLM calculations $(\mathrm{Dc}=\mathrm{yn} / \mathrm{h})$ and measured results from $\mathrm{Ln}(\mathrm{Dm}=\mathrm{Ln} / \mathrm{h})$ has been defined as $\mathrm{E}=|\mathrm{Dc}-\mathrm{Dm}| / \mathrm{Dm}$. The division line shown in Fig. 7 has the averaged $\mathrm{E}$ of 20\%, $5 \%, 11 \%, 15 \%$ and $16 \%$ between YLM and measured results from Ln respectively. The large discrepancy in the trial $\mathrm{C} 1$ is likely to be caused by the maximum velocity not being located at the free surface in this case. Overall, the locations of division lines determined from Ln is similar to YLM (as shown in Fig. 7), especially when $\mathrm{h} / \mathrm{r}$ is equal to 0.67 from Knight and Sterling's (2000) data with the averaged $\mathrm{E}$ only $5 \%$. It can be reasonable explained by YLM as the energy transfer from the main flow region to the boundary is always accompanied with a variation of velocity.

\section{Boundary Shear Stress Distribution}

Some analysis of the boundary shear stress $\tau / \bar{\tau}$ is given by Knight and Sterling (2000), these data will not be commented on this paper. Fig. 8 shows the distribution of boundary shear stress $\tau / \bar{\tau}$ for a circular channel $(\mathrm{C} 1, \mathrm{C} 2$ and $\mathrm{C} 3)$ in a uniform flow for different water depth. It is noted that $\tau$ determined by the local shear velocities ${ }^{u_{*_{s}}}$ were obtained from the velocity distribution for each velocity profile. All of the distributions of $\tau / \bar{\tau}$ in Fig. 8 indicate that the boundary shear stress rises from the corner and approaches a stable value when it is close to either side of the centreline. As the depth of water increases, this stable shear stress extends laterally until eventually all at the shear stress distribution is more or less uniform over the whole boundary. It can be seen 
that there is good agreement between the distributions, indicating a general level of consistency in these data. From these datasets the local boundary shear stress is close to the global value across a large proportion of the channel bed (for instance, $0.75<\operatorname{Pr} / \mathrm{P}<1$ ), which again points to the fact that the distribution of bed shear becomes more uniform as the depth increases. The large variation in the corner regions can be attributed to secondary flow currents transporting momentum away from them along the solid boundaries of the bed and the wall

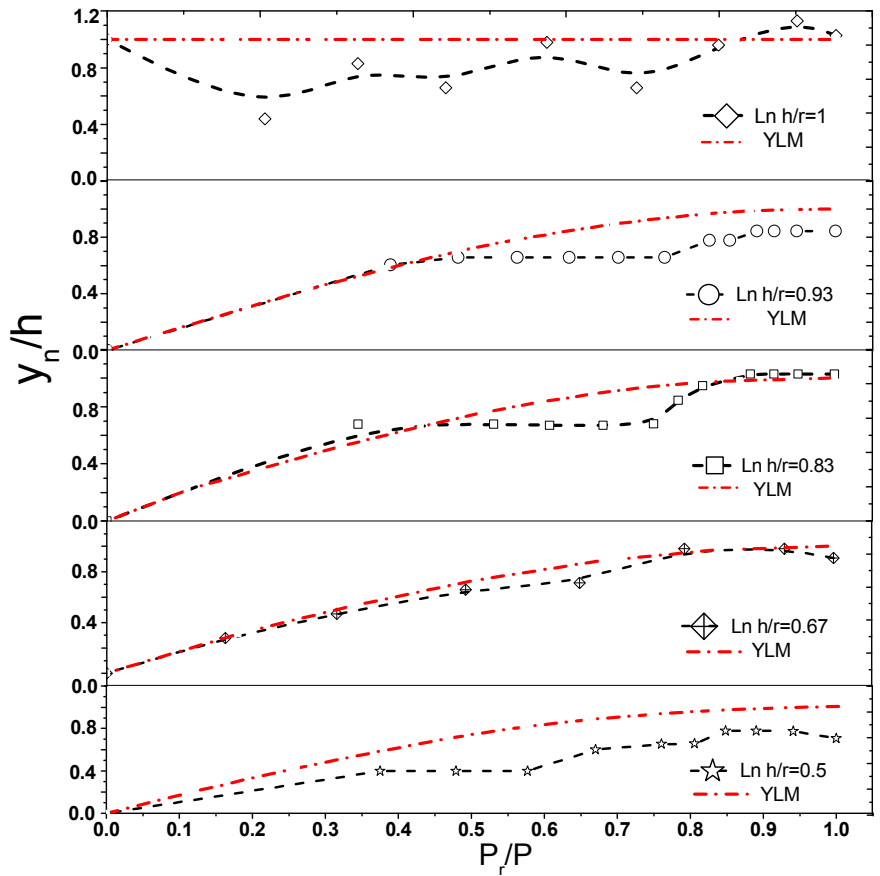

Fig 7. The division lines measured by energy transfer path $L_{n}$ (after experiments data $(\mathrm{C} 1, \mathrm{C} 2$ and $\mathrm{C} 3)$ and Knight and Sterling (2000) data)

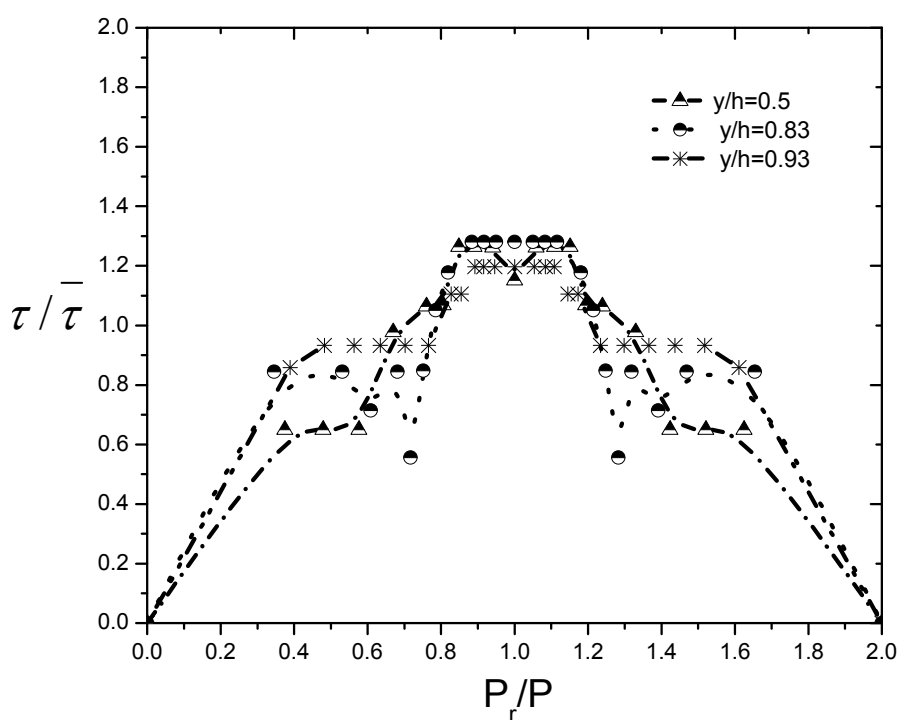

Fig 8. Boundary shear stress distributions with $\mathrm{y} / \mathrm{h}=0.5,0.83$ and 0.93

\section{Conclusions}

This paper investigated whether a 3-D flow region can be divided by analysing the measured mean velocity data. To validate the existence of division lines from the distribution of velocity, a generalised method was developed that can be used to study the shape of the cross sectional flow. The following conclusions can be drawn from this study: 
The experimental method used to test the existence of the division line is to determine the normal distance from a boundary Ln, based on the local boundary shear stress. Using the YLM analytical model, the division line or zero shear stress line should be presented as the free surface line in channel flows. By analysing the velocity data from the literature and our experiments, it is interesting to find that the upper-boundary or division line determined from $\mathrm{Ln}$ is similar to YLM which is closer to the water surface as shown in Fig. 7.

To validate the YLM, the relative error between YLM and measured results has been calculated. The minimum relative error (5\%) existed when the aspect ratio was 0.67 using Knight and Sterling's (2000) data. The range of relative error has been found to be between $5 \%$ to $20 \%$ for the $\mathrm{h} / \mathrm{r}$ ratio between 0.5 to 1 from experiments. This is also a future confirmation of the theory proposed by Yang and his co-authors (Yang and Lim, 1997, Yang, 2005) that the energy transportation occurs through minimum relative distance.

\section{Acknowledgement}

The authors gratefully acknowledge the reviewers' critical comments that greatly improve the paper's readibility. The authors also express gratitude to the financial support from National Natural Science Foundation of China (Grant No. 51509248), Chinese Universities Scientific Fund (Grant No. 2015QC025), National Key Research and Development Plan (Grant No. 2016YFC0400207), Open Research Fund Program of State Key Laboratory of Hydroscience and Engineering of China, Tsinghua University(Grant No.sklhse-2015-C-03),Simulation and Regulation Water Cycle in River Basin, China Institute of Water Resources and Hydropower Resear ch (Grant No. IWHR-SKL-201505).

\section{References}

[1]CARDOSO, A. H., GRAF, W. H. \& GUST, G. 1989. Uniform flow in a smooth open channel. J. Hydraul. Res., 27, 603-616.

CHIEN, N. \& WAN, Z. 1999. Mechanics of Sediment Transport, VA, USA.

[2]DEY, S. 2002. Secondary boundary layer and wall shear for fully developed flow in curved pipes. Proc. R. Soc. Lond., A 458, 283-298.

[3]DEY, S. \& LAMBERT, M. F. 2005. Reynolds stress and bed shear in nonuniform unsteady open-channel flow. J. Hydraul. Eng., 131, 610-614.

[4]EINSTEIN, H. A. 1942. Formulas for the transportation of bed load. Trans. Am. Soc. Civ. Eng., 107, 561-597.

[5]GUO, J. \& JULIEN, P. 2005. Shear stress in smooth rectangular open-channel flows. J. Hydraul. Eng., 131, 30-37.

[6]KEULEGAN, G. H. 1938. Laws of turbulent flow in open channels. J. Res. Natl. Bur. Stand., 21, 708-741.

[7]KNIGHT, D. W. \& STERLING, M. 2000. Boundary shear in circular pipes running partially full. J. Hydraul. Eng., 126, 263-275.

[8]NIKURADSE, J. 1932. Laws of flow in rough pipes Washington DC NACA TM. 1292

[9]SMITS, A. J., MCKEON, B. J. \& MARUSIC, I. 2011. High-reynolds number wall turbulence. Annu. Rev. Fluid. Mech., 43, 353-375.

[10]YANG, S. \& LIM, S. 1997. Mechanism of Energy Transportation and Turbulent Flow in a 3D Channel. J. Hydraul. Eng., 123, 684-692.

[11]YANG, S. Q., LIM, S. Y., MCCORQUODALE, J. A. 2005. Investigation of near wall velocity in 3-D smooth channel flows. J. Hydraulic Res., 43, 149 - 157. 
[12]YANG, S. Q., TAN, S. K. \& WANG, X. K. 2012. Mechanism of secondary currents in open channel flows. J. Geophys. Res., 117, F04014.

[13]YANG, S. Q., XU, W. L., YU, G. L. 2006. Velocity distribution in a gradually accelerating free surface flow. Adv. Water Resour., 29, 1969-1980.

[14]YEN, B. C. 2002. Open channel flow resistance. J. Hydraul. Eng., 128, 20-39.

[15]YOON, J., SUNG, J. \& HO LEE, M. 2012. Velocity profiles and friction coefficients in circular open channels. J. Hydraul. Res., 50, 304-311. 